Agron. Mesoam. 29(2):275-291. Mayo-agosto, 2018

ISSN 2215-3608, doi:10.15517/ma.v29i2.28759

http://www.revistas.ucr.ac.cr/index.php/agromeso

\title{
Crecimiento inicial de Eugenia stipitata, Inga spectabilis e Inga edulis en Napo, Ecuador
}

\section{Initial growth of Eugenia stipitata, Inga spectabilis, and Inga edulis in Napo, Ecuador}

\author{
Ricardo Vinicio Abril-Saltos², Tomás Elías Ruiz-Vázquez³, Jatnel Alonso-Lazo3, \\ Génova Marjorie Cabrera-Murillo ${ }^{4}$, Olivier Angel Meric ${ }^{2}$
}

\begin{abstract}
Resumen
La agricultura ha sido una de las causas de la deforestación, causante de impacto ambiental y degeneración de suelos, lo que conlleva a un menor ingreso percibido por los agricultures. De ahí la necesidad de implementar sistemas agroforestales. Esta investigación tuvo como objetivo describir el crecimiento inicial de Eugenia stipitata, Inga spectabilis e Inga edulis hasta los 320 días desde la emergencia. Se llevó a cabo en el Centro de Investigación, Posgrado y Conservación de la Amazonía de la Universidad Estatal Amazónica, entre 2014-2015. Se evaluó el crecimiento de las especies, para lo cual se registró la altura de la planta, diámetro del tallo, número de hojas y longitud de ramas. Las medidas altura de la planta y diámetro del tallo, se compararon en el tiempo, mediante análisis de varianza. También se comparó si las variaciones en las precipitaciones influyeron en el crecimiento. Los modelos lineal y polinomial, presentaron mejor ajuste en las especies para la altura de la planta y el diámetro del brote. Existió predominancia de hojas verdes sobre amarillas y secas, y junto con el largo de ramas mostraron diferencias significativas en todas las edades de muestreo. El análisis de varianza de doble vía reportó diferencias significativas en la interacción edad y precipitación, sobre la altura de la planta y el diámetro del tallo. Eugenia stipitata tuvo un crecimiento menor que Inga edulis e Inga spectabilis. La producción y crecimiento de ramas, número de hojas fueron también elementos importantes del crecimiento.
\end{abstract}

Palabras clave: sistemas agroforestales, agroforestería, árboles de propósito múltiple, desarrollo agrícola.

\begin{abstract}
Agriculture has been one of the causes of deforestation, causing environmental impact and soil degeneration, which leads to lower income earned by farmers; hence the need to implement agroforestry systems .This research aims to describe from the initial growth of Eugenia stipitata, Inga edulis, and Inga spectabilis to 320 days after emergence. The
\end{abstract}

\footnotetext{
1 Recibido: 4 de mayo, 2017. Aceptado: 28 de setiembre, 2017. Este trabajo formó parte de la tesis del primer autor para obtención del grado de Doctor en Ciencias Agrícolas, en la República de Cuba, bajo auspicio de la Universidad Estatal Amazónica, Ecuador.

2 Universidad Estatal Amazónica, Departamento de Ciencias de la Vida, carrera de Ingeniería Ambiental. Km 2 1⁄2 Vía a Napo, Pastaza, Ecuador.rvabril@uea.edu.ec,americ@uea.edu.ec

3 Instituto de Ciencia Animal, Departamento de Pastos y Forrajes, Carretera Central Km 47 y medio San José de las Lajas, Mayabeque, Cuba. terizv@ica.co.cu,jalonso@ica.co.cu

4 PRAGROS (Productores Agrícolas), Km 2 1⁄2 vía a Tarqui, Pastaza, Ecuador. genova112002@yahoo.com
} 
study took place at the Amazon Research and Conservation Center of the Amazonas State University, during 2014 and 2015. The growth process was evaluated in regards to plant height, stem diameter, number of leaves, and branch length. Plant height and stem diameter were compared over time applying variance analysis. Precipitation was also compared to analyze whether its variations had a direct influence on plant growth. The linear and polynomial models fitted better for thespecies regarding plant height and sprout diameter. Predominance of green leaves compared to yellow and dry ones, as branch length showed significant differences over all the sampling periods. The two-way analysis of variance showed significant differences in the correlation between age and precipitation over plant height and stem diameter. The outcomes led to conclude that Eugenia stipitata had a lower growth than both Inga edulis and Inga spectabilis. Branch production and growth and the number of leaves were also important elements of the growth process.

Keywords: agroforestry systems, agroforestry, multipurpose trees, agricultural development.

\section{Introducción}

La producción agrícola tradicional, ha basado su desarrollo en sistemas que implican la tala total de áreas boscosas e incluso actividades de quema. Esto ha conllevado a un empobrecimiento del suelo, generando consecuencias negativas en la economía del productor y en el ambiente (Proyecto-de-manejo-sostenible-deRecursos-Naturales, 2007). Esta situación ha generado la necesidad de desarrollar sistemas agroforestales, donde la combinación de árboles con cultivos permita mejoras en la producción, diversificación de productos y protección del suelo y el ecosistema (Monro et al., 2016).

La Amazonia ecuatoriana tiene como característica la presencia de bosques, donde la Provincia del Napo presenta un $73 \%$ de su extensión con cobertura de bosque y un 13,2\% de suelos dedicados a producción agropecuaria, con el cacao como uno de los principales cultivos (SENPLADES, 2015). Esto plantea la necesidad de contar con especies que puedan desarrollarse en forma rápida para las condiciones de regiones subtropicales en la Amazonia y que, posteriormente, permitan el desarrollo de sistemas agroforestales con una diversificación de la producción. Las especies del género Inga, han sido reportadas con su uso en este tipo de sistemas, Monro et al. (2016) destacaron en la Amazonía peruana especies de este género con rápido crecimiento y con aporte de nitrógeno y sombra al ecosistema. Inga edulis Mart. es un árbol de copa densa y ancha con altura de hasta $30 \mathrm{~m}$ y diámetro entre 30 y $60 \mathrm{~cm}$. Por otro lado, su fruto es una legumbre de 40 a $180 \mathrm{~cm}$ de longitud, posee en su interior semillas rodeadas por arilos blanquecinos algodonosos (Salazar et al., 2000), reporta usos en alimentación y medicina humana (Ríos et al., 2007), en alimentación animal, material de construcción y con usos ambientales como generador de sombra (Marín et al., 2012). Mientras que Inga spectabilis (Vahl) Willd. es un árbol de hasta $30 \mathrm{~m}$, con diámetro de 20 a $60 \mathrm{~cm}$, su fruto es un arilo comestible, es reportada con usos en alimentación humana (Ríos et al., 2007), además, es utilizada como árbol de sombra en cultivos perennes (café, cacao), producción de miel y sus semillas son alimento animal (Geilfus, 1994; Cordero y Boshier, 2003).

Eugenia stipitata McVaugh, es un arbusto con follaje denso y altura aproximada de $3 \mathrm{~m}$ (Geilfus, 1994); el fruto es una baya globoso-cóncava o esférica, color verde claro, que se torna amarillento o anaranjado en la madurez, pulpa espesa y jugosa, entre amarillo y naranja, aromática y agridulce (Hernández et al., 2007), el cual reporta usos en alimentación (Geilfus, 1994), también medicinales (Medeiros et al., 2013).

Las características de las especies mencionadas, potencializan su uso dentro de sistemas agroforestales, ya que, diversifican la producción, además de ser fuentes generadoras de sombra y fijadoras de nutrientes como es el caso de las especies del género Inga. Las especies evaluadas se encuentran distribuidas en Centro y Sur América, donde se reportan estudios de Eugenia stipitata en regiones de Colombia (Hernández et al., 2007); Inga spectabilis e Inga edulis han sido estudiadas en regiones tropicales de Centroamérica, Colombia, Brasil y Perú (Geilfus, 1994; Marín 
et al., 2012; Cordero y Boshier, 2003). Estos estudios permiten establecer un marco de referencia de su crecimiento y así, compararlo con producciones en un clima subtropical con un bioma de bosque húmedo premontano, como el de la región amazónica ecuatoriana, en donde estas especies crecen en forma silvestre y en cultivo, y actualmente, son comercializadas en mercados locales con una producción en determinadas épocas del año, pero también pueden presentar otros usos para la población y el ecosistema.

El crecimiento vegetal es la consecuencia de la división y la elongación de las células, del proceso de diferenciación o especialización celular (Rodríguez y Lehiner, 2006). Existen factores climáticos (Baruch y Fisher, 1991; Herrera et al., 2006) y de competencia que afectan el desarrollo de la planta, donde la etapa de establecimiento es la más vulnerable, por la baja cantidad de reservas que tiene y la ausencia de un sistema radicular desarrollado (Baruch y Fisher, 1991).

Un crecimiento rápido en las especies vegetales permite captar más recursos (luz, agua y nutrientes), pero se convierten en condiciones adversas cuando existe déficit de los mismos (Villar et al., 2005). Grime (2006) planteó la competición como un factor de incremento cuando uno o más recursos están limitando la productividad; también indicó una clasificación para las especies vegetales de acuerdo con sus características de crecimiento y resistencia, siendo: competitivas si tienen tasas de crecimiento acelerado y baja tolerancia al estrés, tolerantes si tienen bajas tasas de crecimiento, pero mejor tolerancia al estrés, y ruderales cuando presentan resistencia a condiciones adversas y tasas de crecimiento acelerada.

Al representar el crecimiento en función del tiempo, se obtiene una curva, donde se pueden observar tres fases, la primera denominada crecimiento inicial, donde existe poco desarrollo, la segunda denominada fase exponencial, donde se muestra la mayor tasa de crecimiento y la última fase, envejecimiento, donde la planta deja de crecer (Vilchez et al., 2015). El estudio de estos procesos, puede ser representado por métodos de análisis matemático, a través de modelos lineales y no lineales (Ruiz et al., 2013; Rodríguez, 2015) que se ajusten al conjunto de datos experimentales. Los modelos de Gompertz y el logístico son de uso frecuente en el análisis de crecimiento de plantas y animales (Rodríguez, 2015); sin embargo, al utilizar modelos con muchos parámetros hay que valorar si los mismos tienen un significado biológico (Rodríguez y Lehiner, 2006).

Los antecedentes, permiten plantear la hipótesis de que, las especies E. stipitata, I. spectabilis e I. edulis, tienen un rápido crecimiento inicial, que les permite desarrollarse en condiciones de bosque húmedo premontano.

Esta investigación tuvo como objetivo describir el crecimiento inicial de Eugenia stipitata, Inga spectabilis e Inga edulis hasta los 320 días desde la emergencia.

\section{Materiales y métodos}

\section{Zona de estudio}

Los ensayos se desarrollaron entre 2014 y 2015, en una parcela ubicada en el Centro de Investigaciones, Posgrado y Conservación de la Amazonía (CIPCA), de la Universidad Estatal Amazónica, ubicado en los cantones Santa Clara de la provincia de Pastaza y Arosemena Tola de la provincia de Napo, Ecuador, cuyas coordenadas en sistema WGS84 son: 18 M 179053 UTM 9862866, con una altitud de $547 \mathrm{msnm}$ Los reportes históricos indican valores de precipitación anual de $3703 \mathrm{~mm}$ y temperaturas medias entre 18 a $24{ }^{\circ} \mathrm{C}$ (Gobierno-AutónomoDescentralizado-Provincial-de-Pastaza, 2015), su suelo es clasificado como Inceptisol (Gobierno-AutónomoDescentralizado-Provincial-de-Pastaza, 2015) y su clasificación de bioma corresponde a un bosque pluvial premontano (Mariscal, 2016).

Para conocer las condiciones meteorológicas del período de desarrollo, se descargaron los registros de la estación meteorológica automática Pindo Mirador, ubicada a $33 \mathrm{~km}$ del sitio de ensayo, para el período comprendido entre marzo 2014 y junio 2015 , se presentó una temperatura promedio entre 16 a $18{ }^{\circ} \mathrm{C}$, mientras que, 
entre abril 2014 y junio 2015 (Figura 1) los valores de precipitación más altos se reportaron en los meses de enero y marzo del 2015, y los más bajos en septiembre del 2014, para el año 2014 se reportó una media mensual de 359 mm y para el 2015 de $523 \mathrm{~mm}$.

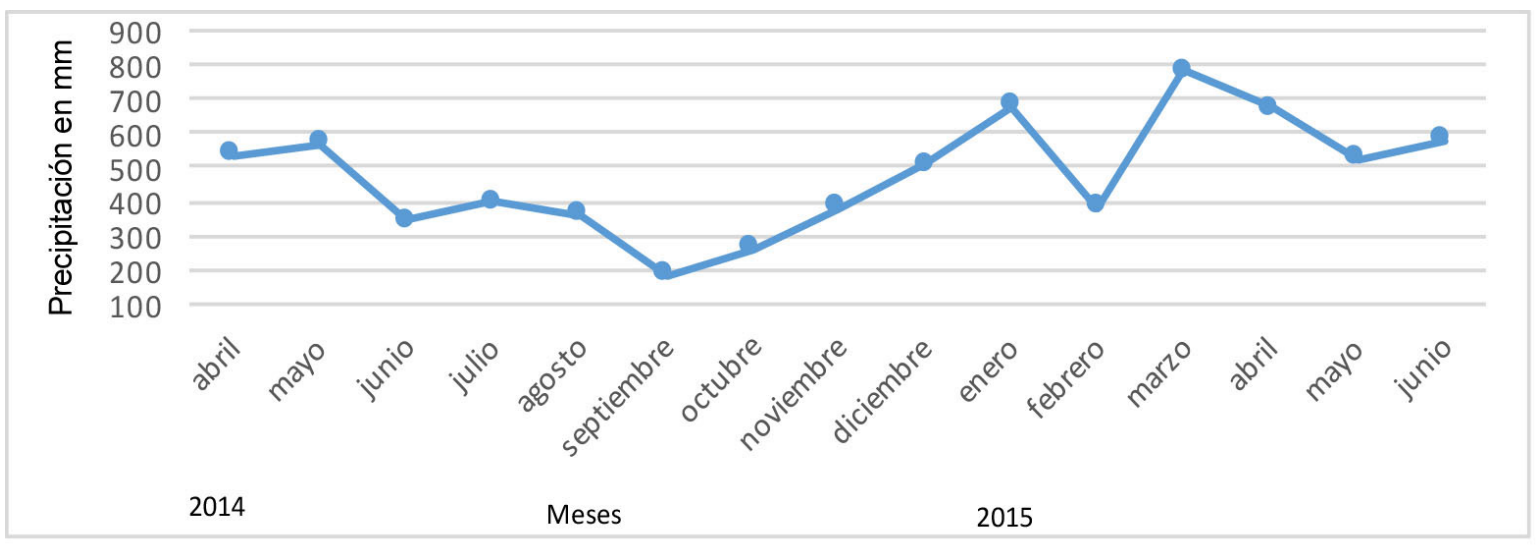

Figura 1. Precipitación acumulada mensual en la estación Pindo Mirador en el período abril 2014 - junio 2015. Mera, Pastaza, Ecuador.

Figure 1. Monthly cumulative precipitation at Biological Station Pindo Mirador from April 2014 to June 2015. Mera, Pastaza, Ecuador.

El lugar donde se desarrolló el ensayo presenta topografía ondulada, formación de bosque de realce, suelo con características de arcilla compacta, drenaje deficiente y pendiente del 5\%. Se utilizaron $1000 \mathrm{~m}^{2}$, área que fue previamente acondicionada de forma manual, con labores que consistieron en desbroce, destronque y deshierbe.

La preparación del suelo se realizó con azadón, en franjas de $12 \mathrm{~m}$ de ancho y $18 \mathrm{~m}$ de largo. Los surcos se establecieron a 2,5 m de separación, con $10 \mathrm{~m}$ de largo y se repitieron cinco veces. La distancia entre plantas para todas las especies fue de $1 \mathrm{~m}$. Se sembraron diez plantas por surco para un total de cincuenta plantas por especie; se tomaron las medidas de crecimiento en diez individuos por especie. Los surcos de cada especie se distribuyeron al azar en el área del ensayo.

Las semillas para la siembra se obtuvieron de frutos maduros de plantas silvestres ubicadas en la misma zona, se verificó que estuvieron completamente desarrolladas. En función de la época de producción de frutos y disposición de semillas, se realizó la siembra directa en orificios en los surcos, con una profundidad aproximada de 8 cm. I. spectabilis se sembró en junio del 2014, E. stipitata en julio del 2014 e I. edulis en septiembre del 2014.

Las observaciones se realizaron cada treinta días hasta los 300 días, y la última medición a los 320 días desde la emergencia. En cada planta se midió la altura $(\mathrm{cm})$, diámetro del tallo $(\mathrm{mm})$, número total de hojas, largo promedio de las ramas $(\mathrm{cm})$.

La altura se midió con una cinta métrica, desde la superficie del suelo hasta la yema apical. El diámetro de la planta se obtuvo con un pie de rey (con 0,05 mm de precisión), a ras del suelo. En cada planta se contó el número de hojas verdes amarillas, a aquellas que poseían más del 50\% de su área foliar de color amarillo, lo cual indicaría signos de senescencia (Díaz-Maruto et al., 1993), y completamente secas, cuando estas se desprendían del tallo. La longitud promedio de las ramas en cada especie se midió en tres ramas al azar por planta, desde el tronco central hasta la yema final de la rama con cinta métrica. 


\section{Análisis estadístico}

Se utilizó el programa Table Curve 2D (Systat, 2002) para determinar los modelos más adecuados de crecimiento para las variables altura de la planta y diámetro del tallo. También se aplicó el modelo Gompertz a través del programa Statgraphics (Statpoint, 2005). La selección del modelo, para cada caso, se realizó según los criterios establecidos por Díaz et al. (2014), y se escogieron aquellos que fueron significativos en P>F, presentaron mayor coeficiente de determinación (R2), menor error experimental y significación de los parámetros del modelo (Cuadro 1). A estos criterios, y dado los intereses de la presente investigación, se incorporó la simplicidad del modelo como elemento para su elección, lo cual también pretende dar un significado biológico del mismo (DiBenedetto y Tognetti, 2016). En las ecuaciones analizadas, la variable "X" corresponde a la edad de la planta en días y la variable "Y" a la altura de la planta o diámetro del tallo o brote. Se midió la altura de la planta y diámetro del tallo respecto a la precipitación acumulada en cada edad de muestreo, a través de un análisis de varianza de dos vías, con el programa SPSS 22 (IBM, 2013), donde se comparó las precipitaciones acumuladas y la edad, para establecer efectos en el crecimiento de las medidas indicadas.

Para las variables número total de hojas y largo de la rama, se realizó análisis de varianza y se aplicó la prueba a posteriori de Duncan (1955) para la comparación de medias. Se empleó el paquete estadístico INFOSTAT versión 2014 (Di-Rienzo et al., 2014), y los valores de número de hojas totales se transformaron con la función raíz cuadrada, con el fin de reducir anomalías tales como, la no aditividad, la no normalidad y la heterocedasticidad (Herrera, 2013). El análisis de las hojas verdes, amarillas y secas se realizó en función del porcentaje.

\section{Resultados}

Se encontró crecimiento continuo de las plantas durante el período de evaluación (Figura 2). Los modelos matemáticos evaluados (Cuadro 2) para describir este comportamiento, mostraron su mejor ajuste en modelos lineales y polinómicos. En E. stipitata la altura se incrementó de forma constante (0,089 cm/día) (Figura 2A); la misma no presentó asíntota al final del período de evaluación, lo que indica que continuó en fase de crecimiento vegetativo.

En I. spectabilis (Figura 2B), se observó una tasa de crecimiento de $0,23 \mathrm{~cm} /$ día, y se mantuvo hasta los 270 días, donde se incrementó a $0,39 \mathrm{~cm} /$ día, hasta los 300 días, para posteriormente presentar su mayor crecimiento con una tasa de $0,42 \mathrm{~cm} /$ día, lo cual muestra que a partir de este momento la especie entró en su etapa de desarrollo más acelerado. Si bien el modelo de mejor ajuste fue el exponencial, el parámetro "a", presentó valores no significativos en el valor de p, por lo cual no fue considerado este modelo. En I. edulis (Figura 2C) el crecimiento fue lento, con una tasa de $0,13 \mathrm{~cm} /$ día, hasta los 270 días, donde incrementó a $0,26 \mathrm{~cm} /$ día y, a partir de los 300 días, presentó su desarrollo más acelerado con una tasa de $0,28 \mathrm{~cm} /$ día, alcanzando una altura máxima de 59,15 $\mathrm{cm}$ al final del período de evaluación sin asíntota.

De los modelos matemáticos evaluados para el crecimiento del diámetro del tallo (Cuadro 2), E. stipitata presentó un crecimiento lineal, con una tasa de $0,089 \mathrm{~mm} /$ día, siendo el más lento de las tres especies (Figura 3A). En I. edulis e I. spectabilis fueron los modelos polinomiales los de mejor ajuste. En I. edulis (Figura 3C) durante los primeros 150 días fue lento, con una tasa de $0,016 \mathrm{~mm} /$ día, posterior a este período, aceleró su crecimiento alcanzando una tasa de $0,042 \mathrm{~mm} /$ día, la cual se mantuvo hasta los 240 días, a partir de los cuales alcanzó su mayor velocidad de crecimiento con una tasa de $0,062 \mathrm{~mm} /$ día. I. spectabilis (Figura 3B) presentó hasta los 240 días una tasa de crecimiento de $0,036 \mathrm{~mm} /$ día y, a partir de este período, mostró una tasa de $0,072 \mathrm{~mm} /$ día, siendo la etapa 
Cuadro 1. Modelos evaluados para crecimiento en altura de E. stipitata, I. spectabilis e I. edulis, en el cantón Arosemena Tola de la provincia de Napo, Ecuador, en el periodo 2014 y 2015.

Table 1. Models evaluated for plant height growth of E. stipitata, I. spectabilis, and I. edulis. in Arosemena Tola, Napo province, Ecuador, from 2014 to 2015.

\begin{tabular}{|c|c|c|c|c|c|c|c|c|c|}
\hline & \multirow[t]{2}{*}{ Modelo } & \multirow{2}{*}{$\begin{array}{c}\text { Signif. } \\
P>F\end{array}$} & \multirow[t]{2}{*}{$\mathbf{R}^{2}$} & \multirow{2}{*}{$\begin{array}{l}\mathbf{E E} \\
( \pm)\end{array}$} & \multicolumn{5}{|c|}{ Significación de los parámetros } \\
\hline & & & & & Criterio & $\mathbf{a}$ & $\mathbf{b}$ & $\mathbf{c}$ & d \\
\hline \multirow{18}{*}{ 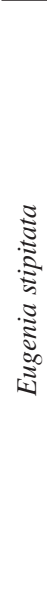 } & Gompertz & $<0,001 * * *$ & 0,98 & 1,47 & Valor estimado & 81,25 & 1,74 & 003 & \\
\hline & & & & & $\mathrm{EE}( \pm)$ & 28,99 & 1,74 & 0,003 & \\
\hline & & & & & Valor de P & $0,024 *$ & $0,0004 * * *$ & $0,0227 *$ & \\
\hline & Logístico & $<0,001 * * *$ & 0,98 & 1,49 & Valor estimado & 59,15 & 3,1 & 0,006 & \\
\hline & & & & & $\mathrm{EE}( \pm)$ & 10,33 & 0,58 & 0,001 & \\
\hline & & & & & Valor de $\mathrm{P}$ & $0,0003 * * *$ & $0,0005 * * *$ & $0,0006 * * *$ & \\
\hline & Exponencial & $<0,001 * * *$ & 0,97 & 1,88 & Valor estimado & 16,36 &, 003 & & \\
\hline & & & & & $\mathrm{EE}( \pm)$ & 0,79 & ,0002 & & \\
\hline & & & & & Valor de P & $<0,0001 * * *$ & $<, 0001 * * *$ & & \\
\hline & Lineal & $<0,001 * * *$ & 0,98 & 1,34 & Valor estimado & 13,89 & 0,09 & & \\
\hline & & & & & $\mathbf{E E}( \pm)$ & 0,68 & 0,004 & & \\
\hline & & & & & Valor de $\mathbf{P}$ & $<0,0001 * * *$ & $<0,0001 * * *$ & & \\
\hline & Cuadrático & $<0,001 * * *$ & 0,92 & 2,89 & Valor estimado & 18,6 & 0,0003 & & \\
\hline & & & & & $\mathrm{EE}( \pm)$ & 1,16 & 0,00003 & & \\
\hline & & & & & Valor de $\mathbf{P}$ & $<0,0001 * * *$ & $<0,0001 * * *$ & & \\
\hline & Cúbico & $<0,001 * * *$ & 0,98 & 1,49 & Valor estimado & 14,24 & 0,008 & 0,00009 & 0,0000002 \\
\hline & & & & & $\mathrm{EE}( \pm)$ & 1,19 & 0,04 & 0,0003 & 0,000001 \\
\hline & & & & & Valor de $\mathbf{P}$ & $<0,0001 * * *$ & 0,059 & 0,75 & 0,779 \\
\hline \multirow{18}{*}{ 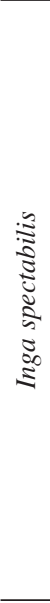 } & Gompertz & $<0,001 * * *$ & 0,95 & 5,62 & Valor estimado & 168,18 & 3,895 & 0,005 & \\
\hline & & & & & $\mathrm{EE}( \pm)$ & 91,019 & 0,433 & 0,002 & \\
\hline & & & & & Valor de $\mathrm{P}$ & 0,819 & 0,1049 & 0,2129 & \\
\hline & Logístico & $<0,001 * * *$ & 0,99 & 1,88 & Valor estimado & 522,76 & 58,63 & 0,008 & \\
\hline & & & & & $\mathrm{EE}( \pm)$ & 554,8 & 58,75 & 0,0009 & \\
\hline & & & & & Valor de P & ns & ns & $0,0095 * *$ & \\
\hline & Exponencial & $<0,001 * * *$ & 0,99 & 2.09 & Valor estimado & 5,542 & 6.76 & -129.17 & \\
\hline & & & & & $\mathrm{EE}( \pm)$ & 2.74 & 1.65 & 11.68 & \\
\hline & & & & & Valor de P & 0.7363 & $0.0273 * *$ & $<0.0001 * * *$ & \\
\hline & Lineal & $<0,001 * * *$ & 0,92 & 7,43 & Valor estimado & $-4,288$ & 0,245 & & \\
\hline & & & & & $\mathrm{EE}( \pm)$ & 4,824 & 0,024 & & \\
\hline & & & & & Valor de $\mathrm{P}$ & 0,3976 & 0 & & \\
\hline & Cuadrático & $<0,001 * * *$ & 0,98 & 3.42 & Valor estimado & 12,3 & 0,0007 & & \\
\hline & & & & & $\mathbf{E E}( \pm)$ & 1,46 & 0,00003 & & \\
\hline & & & & & Valor de $\mathbf{P}$ & $<0,0001 * * * * *$ & $<0,0001 * * *$ & & \\
\hline & Cúbico & $<0,001 * * *$ & 0,98 & 3.74 & Valor estimado & 17,31 & $0,000002 * * *$ & & \\
\hline & & & & & $\mathrm{EE}( \pm)$ & 1,43 & 0,00001 & & \\
\hline & & & & & Valor de P & $<0,0001 * * *$ & $<0,0001 * * *$ & & \\
\hline \multirow{18}{*}{ 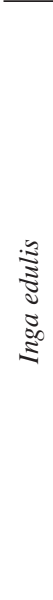 } & Gompertz & $<0,001 * * *$ & 0,97 & 3,16 & Valor estimado & 146,48 & 3,23 & 0,0039 & \\
\hline & & & & & $\mathrm{EE}( \pm)$ & 85,2 & 0,41 & 0,0015 & \\
\hline & & & & & Valor de P & 0,1365 & $<0,0001 * * *$ & $0,0368^{*}$ & \\
\hline & Logístico & $<0,001 * * *$ & 0,97 & 3,34 & Valor estimado & 82,44 & 11,99 & 0,01 & \\
\hline & & & & & $\mathrm{EE}( \pm)$ & 21,03 & 2,53 & 0,002 & \\
\hline & & & & & Valor de P & $0,0044 * *$ & $0,0008 * * *$ & $0,0009 * * *$ & \\
\hline & Exponencial & $<0,001 * * *$ & 0,98 & 2,21 & Valor estimado & 10,121 & $-182,70$ & & \\
\hline & & & & & $\mathrm{EE}( \pm)$ & 0,753 & 9,387 & & \\
\hline & & & & & Valor de $\mathrm{P}$ & $<0,0001 * * *$ & $<0,0001 * * *$ & & \\
\hline & Lineal & $<0,001 * * *$ & 0,97 & 3,32 & Valor estimado & 0,844 & 0,165 & & \\
\hline & & & & & $\mathrm{EE}( \pm)$ & 1,813 & 0,009 & & \\
\hline & & & & & Valor de P & 0,6513 & $0,00000 * * *$ & & \\
\hline & Cuadrático & $<0,001 * * *$ & 0,98 & 2,17 & Valor estimado & 11,84 & 0,000046 & & \\
\hline & & & & & EE $( \pm)$ & 1,02 & 0,000002 & & \\
\hline & & & & & Valor de $\mathbf{P}$ & $<0,0001 * * *$ & $<0,0001 * * *$ & & \\
\hline & Cúbico & $<0,001 * * *$ & 0,98 & 2,44 & Valor estimado & 10,153 & 0,0007 & 0,000001 & \\
\hline & & & & & $\mathrm{EE}( \pm)$ & 1,541 & 0,00013 & 0,0000003 & \\
\hline & & & & & Valor de $\mathrm{P}$ & $0,0001 * * *$ & $0,00075 * * *$ & 0,1462 & \\
\hline
\end{tabular}

Significación: $0,01 *, 0,05 * *, 0,001 * * * /$ Significant: $0.01 *, 0.05 * *, 0.001 * * *$. 

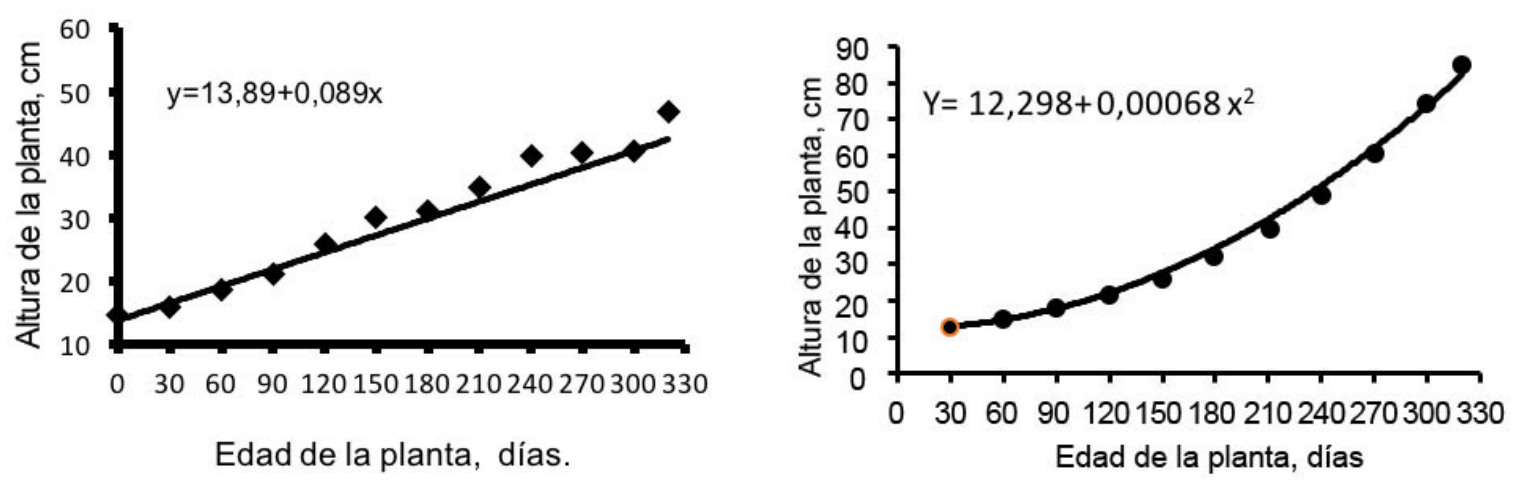

Edad de la planta, días.

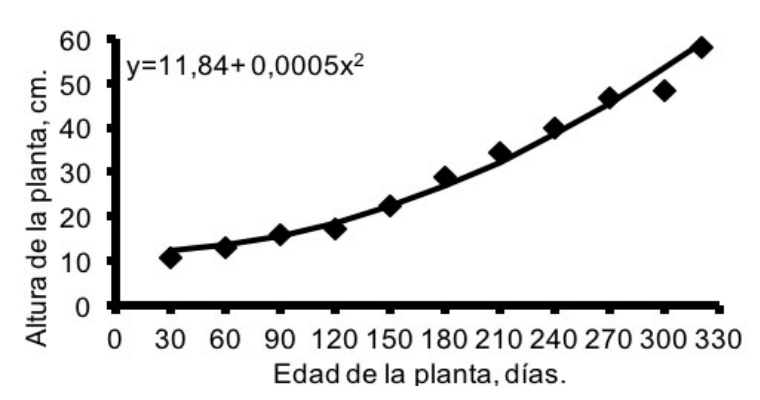

Figura 2. Crecimiento de la altura de la planta $(\mathrm{cm})$. A. E. stipitata, B. I. spectabilis y C. I. edulis, en el cantón Arosemena Tola de la provincia de Napo, Ecuador, periodo 2014-2015.

Figure 2. Plant height growth (cm). A. E. stipitata, B. I. spectabilis, and C. I. edulis, in Arosemena Tola, Napo province, Ecuador, from 2014 to 2015 .

de desarrollo más acelerado en el diámetro del tallo. A los 320 días E. stipitata e I. spectabilis (Figura 3A y 3B) alcanzaron 7,98 y 15,81 mm de diámetro respectivamente, mientras que, I. edulis (Figura 3C) alcanzó un diámetro del tallo de $12,73 \mathrm{~mm}$.

El análisis de varianza de dos vías, considerando a las variables precipitación y edad en el crecimiento de las medidas altura de la planta y diámetro del tallo (Cuadro 3), mostró valores significativos al 0,001 en todas las especies para ambas medidas, lo cual indica la incidencia de estas en el crecimiento de las especies.

En E. stipitata, el análisis de dos vías mostró valores significativos $(\mathrm{p}<0,001)$ para la altura de la planta $(\mathrm{mm})$, y el diámetro del tallo (mm) (Cuadro 3, Figura 4) ambos valores se ajustaron mejor a los modelos lineales. Se observó un crecimiento constante en ambas medidas, sin cambios en el patrón de crecimiento, esto demostraría que la variación en las precipitaciones las precipitaciones no generó cambios en el crecimiento.

En I. spectabilis, el análisis de dos vías mostró valores significativos ( $\mathrm{p}<0,001)$ para la altura de la planta (mm), y el diámetro del tallo (mm) (Cuadro 3, Figura 5). El mayor pico de precipitación fue a los 240 días de edad de la planta, lo cual puede influir en el incremento de la tasa de crecimiento en la altura de la planta, donde hasta los 270 días presentó una tasa de $0,23 \mathrm{~cm} /$ día y posteriormente, su tasa de crecimiento aumentó en un $70 \%$, y a partir de los 300 días en un $87 \%$ con respecto a la tasa de crecimiento inicial. En el diámetro del tallo, previo a los 240 días se tuvo una tasa de crecimiento de $0,016 \mathrm{~mm} /$ día y posterior a este período se incrementó en un $162 \%$. Esto puede indicar que el incremento de la precipitación tuvo incidencia en el incremento de la tasa de crecimiento para ambas medidas.

Para I. edulis, el análisis de dos vías mostró valores significativos $(\mathrm{p}<0,001)$ para la altura de la planta $(\mathrm{mm})$ y el diámetro del tallo (mm) (Cuadro 3, Figura 6). Hasta los 270 días presentó una tasa de crecimiento de $0,13 \mathrm{~cm} /$ 
Cuadro 2. Modelos evaluados para crecimiento de diámetro del tallo de E. stipitata, I. spectabilis e I. edulis, en el cantón Arosemena Tola de la provincia de Napo, Ecuador, en el periodo 2014 y 2015.

Table 2. Models evaluated for stem diameter growth of E. stipitata, I. spectabilis, and I. edulis, in Arosemena Tola, Napo province, Ecuador, from 2014 to 2015 .

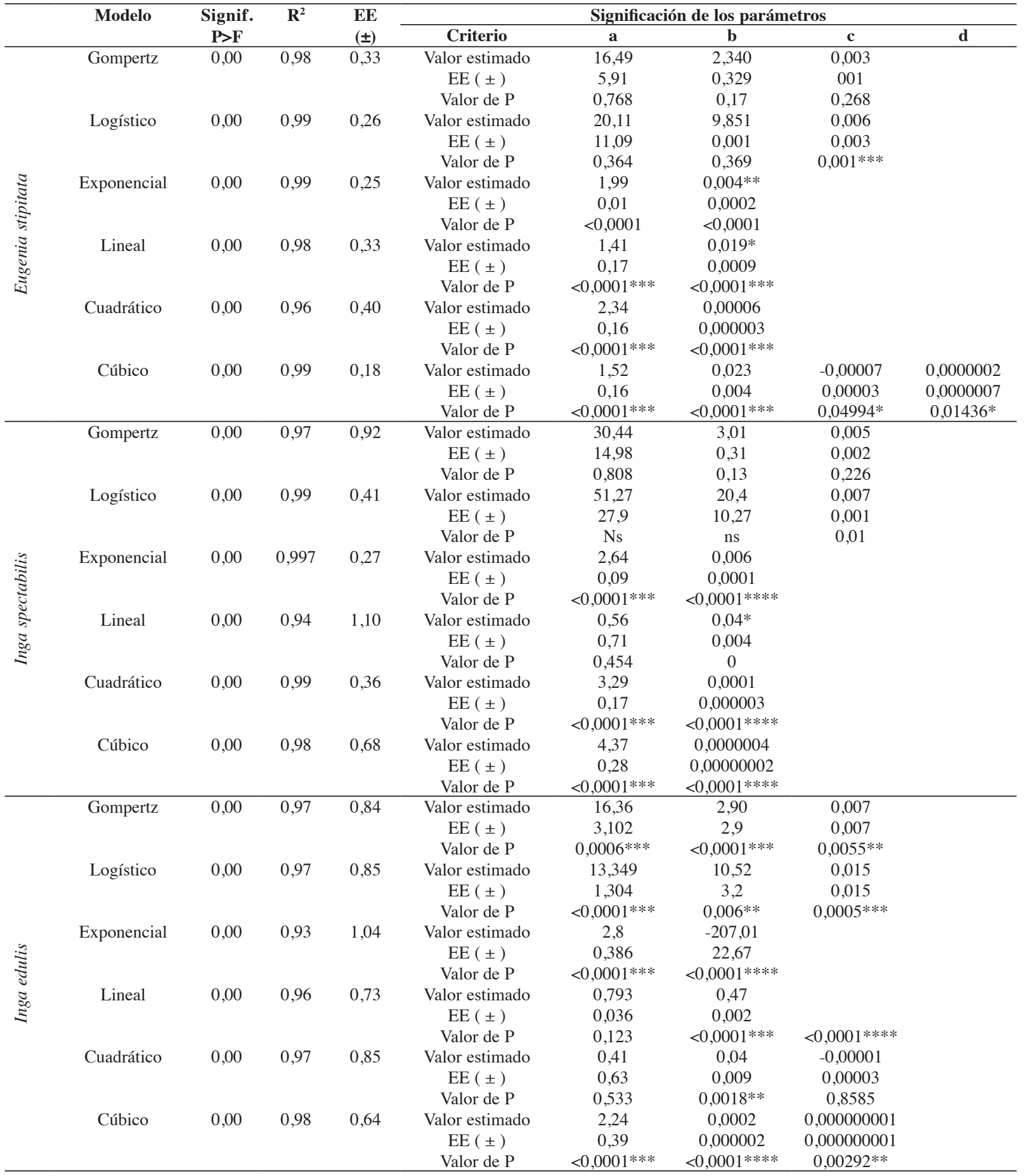

Significancia: $0,01 *, 0,05^{* *}, 0,001 * * * /$ Significant: $0.01 *, 0.05 * *, 0.001 * * *$. 

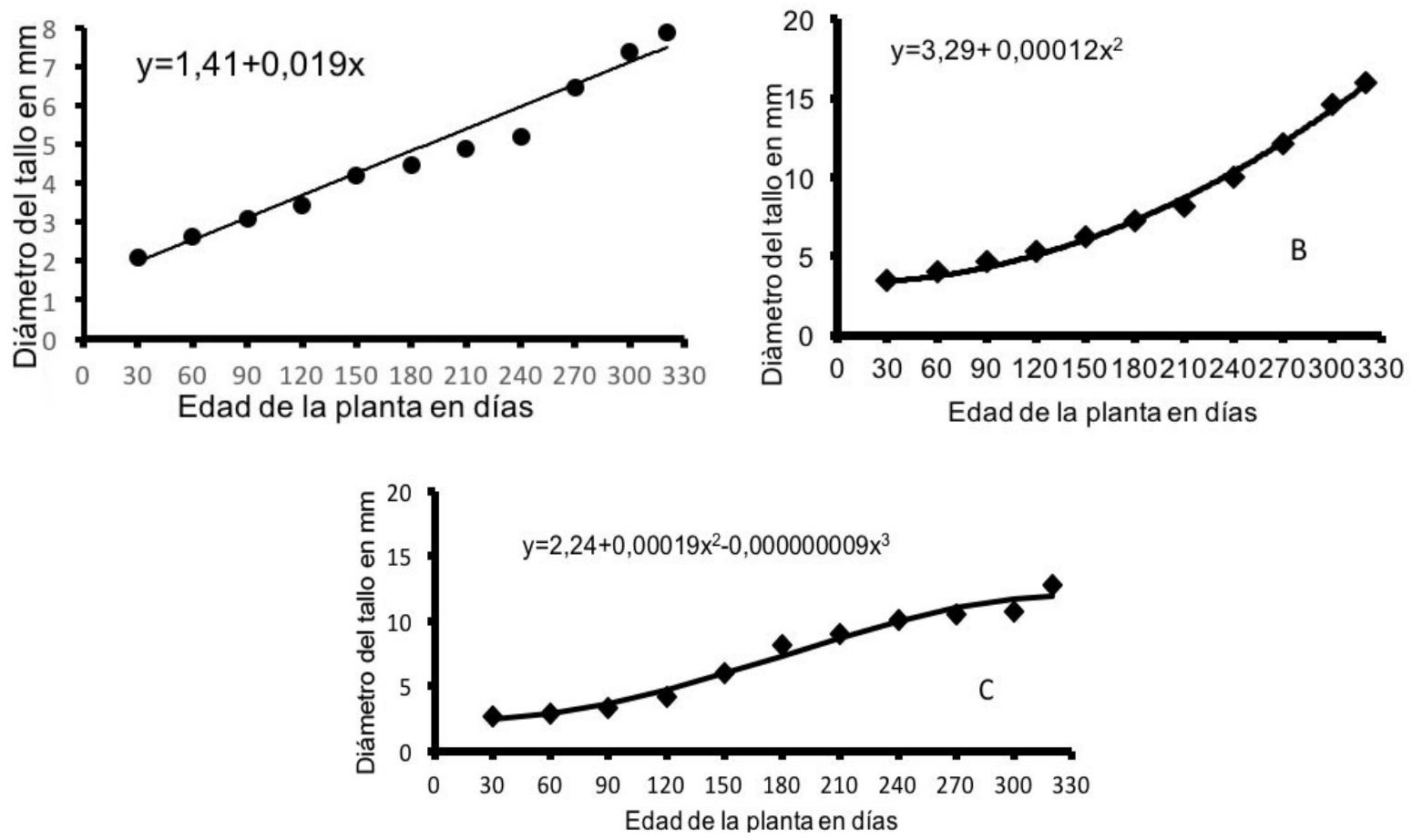

Figura 3. Crecimiento del diámetro del tallo (mm) en. A. E. stipitata, B. I. spectabilis y C. I. edulis, en el cantón Arosemena Tola de la provincia de Napo, Ecuador, en el periodo 2014 y 2015

Figure 3. Stem diameter growth $(\mathrm{mm})$ in: A. E. stipitata, B. I. spectabilis, and C. I. edulis, in Arosemena Tola, Napo province, Ecuador, from 2014 to 2015.

Cuadro 3. Análisis de varianza de dos vías (edad de la planta y precipitación) sobre las medidas altura de la planta y diámetro del tallo de E. stipitata, I. spectabilis e I. edulis, en el cantón Arosemena Tola de la provincia de Napo, Ecuador, en el periodo 2014 y 2015.

Table 3. Two-way analysis of variance (plant age and precipitation) on plant height and stem diameter of E. stipitata, I. spectabilis, and I. edulis, in Arosemena Tola, Napo province, Ecuador, from 2014 to 2015.

\begin{tabular}{lccccccc}
\hline Especie & Medida & $\mathbf{R}^{\mathbf{2}}$ & $\begin{array}{c}\text { Tipo II de suma } \\
\text { de cuadrados }\end{array}$ & gl & $\begin{array}{c}\text { Cuadrático } \\
\text { promedio }\end{array}$ & F & Sig. \\
\hline E. stipitata & altura & 0,63 & 852771,71 & 11 & 77524,70 & 16,80 & $<, 0001 * * *$ \\
& diámetro & 0,72 & 320,32 & 11 & 29,120 & 24,62 & $<, 0001 * * *$ \\
\hline I. edulis & altura & 0,46 & 7955345,45 & 11 & 723213,22 & 19,20 & $<, 0001 * * *$ \\
& diámetro & 0,55 & 3628,74 & 11 & 329,89 & 28,21 & $<, 0001 * * *$ \\
\hline I. spectabilis & altura & 0,67 & 6742892,45 & 11 & 612990,22 & 19,57 & $<, 0001 * * *$ \\
& diámetro & 0,70 & 1710,18 & 11 & 155,47 & 22,46 & $<, 0001 * * *$ \\
\hline
\end{tabular}

*** Significativo al 0,001/*** Significant to 0.001 . 


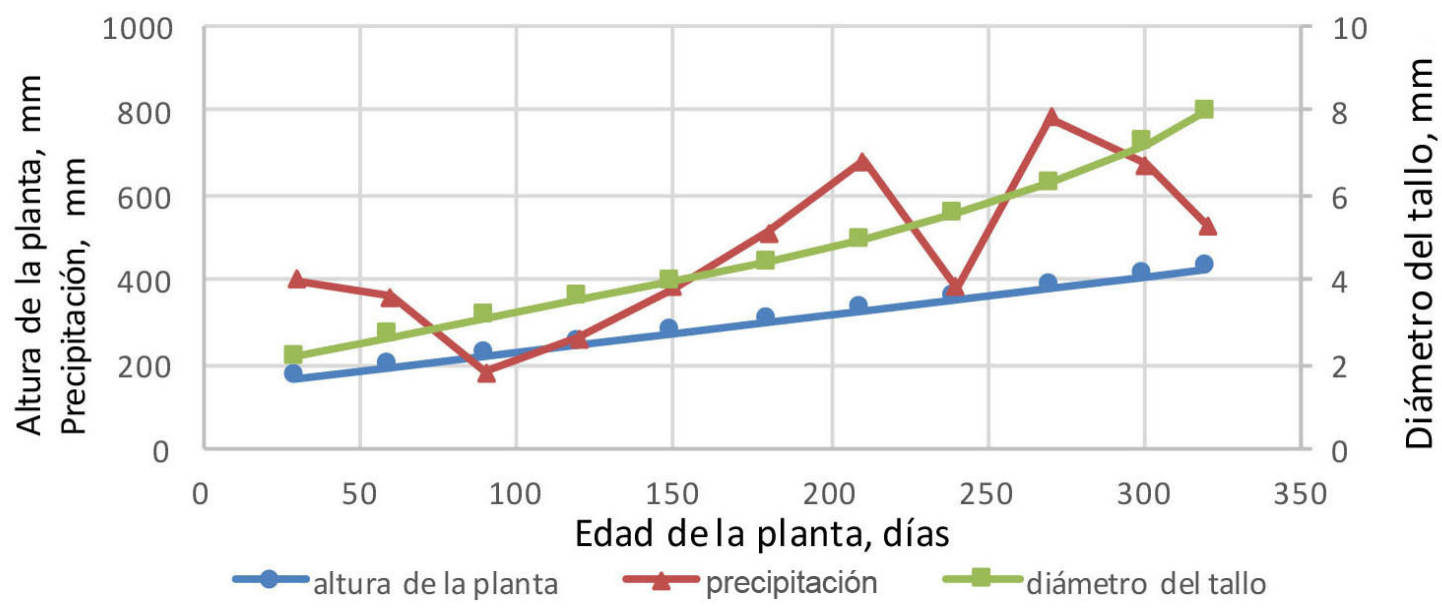

Figura 4. Crecimiento de altura de la planta y diámetro del tallo con la precipitación acumulada en cada período para E. stipitata el cantón Arosemena Tola de la provincia de Napo, Ecuador, en el periodo 2014 y 2015.

Figure 4. Plant height and stem diameter growth correlated with cumulative precipitation in each period for the species E. stipitata, in Arosemena Tola, Napo province, Ecuador, from 2014 to 2015.

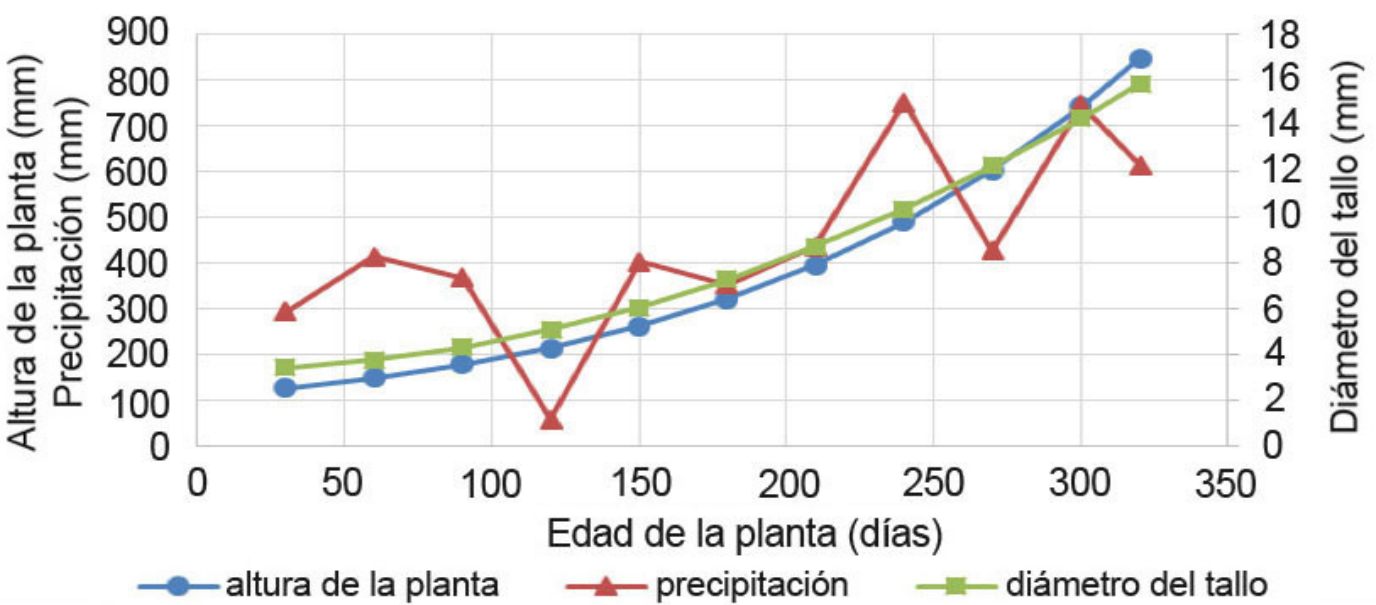

Figura 5. Crecimiento de altura de la planta y diámetro del brote con la precipitación acumulada en cada período para I. spectabilis, el cantón Arosemena Tola de la provincia de Napo, Ecuador, en el periodo 2014 y 2015.

Figure 5. Plant height and sprout diameter growth correlated with cumulative precipitation in each period for the species $I$. spectabilis, in Arosemena Tola, Napo province, Ecuador, from 2014 to2015.

día, a partir de este período se duplicó y alcanzó su máxima con $0,26 \mathrm{~cm} /$ día, y a partir de los 300 días presentó su desarrollo más acelerado con una tasa de crecimiento superior en un $107 \%$ a la tasa inicial. En el crecimiento del diámetro del tallo, presentó una tasa de $0,016 \mathrm{~mm} /$ día hasta los 150 días, luego se incrementó en un 163\%, y a los 240 días fue de un $288 \%$ con respecto a la tasa inicial. En este período las precipitaciones comenzaron a disminuir a partir de los 240 días, con un potencial efecto en el aumento de la tasa de crecimiento en ambas medidas. 


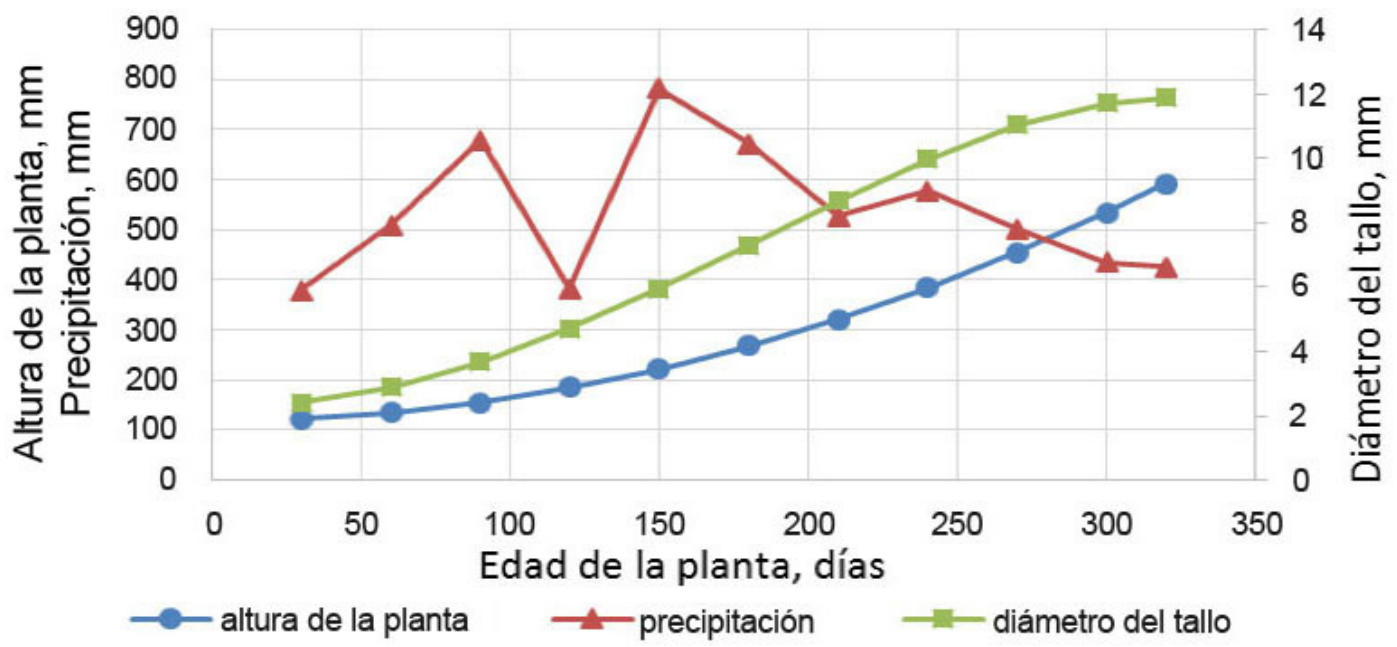

Figura 6. Crecimiento de la altura de la planta y diámetro del brote con la precipitación acumulada en cada período para la especie I. edulis, en el cantón Arosemena Tola de la provincia de Napo, Ecuador, en el periodo 2014 y 2015

Figure 6. Plant height and sprout diameter growth correlated with cumulative precipitation in each period for the species I. edulis, in Arosemena Tola, Napo province, Ecuador, from 2014 to 2015.

En ambas especies del género Inga, los incrementos en las tasas de crecimiento para el diámetro del tallo, se dieron a partir de los 240 días de edad, pero con condiciones de precipitación diferentes, lo cual indicaría que para estas dos especies su mayor tasa de crecimiento en la fase inicial para el diámetro del tallo, comenzó a partir de esa edad, mientras que, para la medida altura de la planta se dio entre los 270 y 300 días de edad. Estas características, pueden representar una ventaja en etapas de instalación en medios naturales o sistemas agroforestales de estas especies.

El número total de hojas (Cuadro 4), presentó cambios significativos a partir de los 30 días, al diferir todas sus medias, y su máximo fue a los 300 días con media de 131 hojas. Existió predominancia de las hojas verdes sobre las amarillas y secas.

Los primeros brotes laterales en E. stipitata comenzaron a activarse a los quince días después de la emergencia, y a los treinta días presentaron una longitud promedio de $3,13 \mathrm{~cm}$, a partir de esa edad se presentaron diferencias estadísticas en cuanto a su longitud, con respecto al resto de edades de registro (Cuadro 5).

El número total de hojas por planta (Cuadro 6), presentó su valor mínimo a los 30 días con media de 4,71, y mostró diferencias significativas a los 120, 210 y 300 días, cuando alcanzó su mayor valor en este período de muestreo con una media de 18,3 hojas. En las hojas verdes, amarillas y secas no hubo diferencias apreciables, aunque existió mayor proporción de hojas verdes en todos los períodos evaluados.

En la longitud de ramas (Cuadro 7), las yemas comenzaron a brotar a partir de los 15 días, y a los 30 días tuvieron media de $3,11 \mathrm{~cm}$ y presentaron diferencias a los 210 y 300 días. En la última edad de muestreo esta medida alcanzó promedio de $20,75 \mathrm{~cm}$.

En el total de hojas por planta (Cuadro 8), I. edulis tuvo su mínimo valor a los 30 días con media de 6,02, no presentó diferencias entre las edades de muestreo hasta los 210 días, y alcanzó su mayor valor, con una media de 22,93 hojas a los 300 días. Existió predominancia de hojas verdes, manteniéndose con valores superiores al 95\%, a excepción de los 300 días, donde entre hojas amarillas y secas alcanzaron un valor de 8,46\%. 
Cuadro 4. Número de hojas totales y proporción de hojas verdes, amarillas y secas de la especie E. stipitata, en el cantón Arosemena Tola de la provincia de Napo, Ecuador, en el periodo 2014 y 2015.

Table 4. Total number of leaves and proportion of green, yellow, and dry leaves of the species E. stipitata, in Arosemena Tola, Napo province, Ecuador, from 2014 to 2015.

\begin{tabular}{ccccc}
\hline \multirow{2}{*}{$\begin{array}{c}\text { Días desde la } \\
\text { emergencia }\end{array}$} & $\begin{array}{c}\text { Número total de hojas } \\
\text { promedio* }\end{array}$ & Verdes & Porcentaje de hojas (\%) \\
\cline { 3 - 5 } & $3,99 \mathrm{a}(16,2)$ & 100 & 0 & Secas \\
\hline 30 & $7,06 \mathrm{~b}(52,7)$ & 100 & 0 & 0 \\
120 & $9,62 \mathrm{c}(95,14)$ & 100 & 0 & 0 \\
210 & $11,34 \mathrm{~d}(131,00)$ & 98 & 0 & 2 \\
\hline
\end{tabular}

Valor de $\mathbf{P}<0,0001 \quad$ EE ( \pm ) 0,55

* Valores entre paréntesis son el promedio de número de hojas por conteo. Datos transformados según $\sqrt{ } \mathrm{X}$ para número total de hojas. $\mathrm{a}, \mathrm{b}, \mathrm{c}, \mathrm{d}$ Valores con letras comunes no difieren para $\mathrm{P}<0,05$ (Duncan, 1955) / Values in parentheses are the average number of leaves per count. Data transformed according to $\sqrt{ } \mathrm{X}$ for total number of leaves. a, b, c, d Values with common letters do not differ for $\mathrm{P}<0.05$ (Duncan, 1955).

Cuadro 5. Longitud de ramas en la especie E. stipitata, en el cantón Arosemena Tola de la provincia de Napo, Ecuador, en el periodo 2014 y 2015.

Table 5. Branch length of E. stipitata, in Arosemena Tola, Napo province, Ecuador, from 2014 to 2015.

\begin{tabular}{|c|c|}
\hline Días desde la emergencia & Longitud promedio de ramas $(\mathrm{cm})$ \\
\hline 30 & 3,13 a \\
\hline 120 & $10,02 \quad b$ \\
\hline 210 & $19,00 \quad \mathrm{c}$ \\
\hline 300 & $28,17 \mathrm{~d}$ \\
\hline \multicolumn{2}{|c|}{ Valor de $\mathrm{P}<0,0001 \quad \mathrm{EE}( \pm) 2,09$} \\
\hline
\end{tabular}

a, b, c, d Valores con letras comunes no difieren para $\mathrm{P}<0,05$ (Duncan, 1955) / Values with common letters do not differ for $\mathrm{P}<0.05$ (Duncan, 1955).

Cuadro 6. Número total de hojas por planta, proporción de hojas verdes, amarillas y secas de la especie I. spectabilis, en los cantones Santa Clara de la provincia de Pastaza y Arrosemena Tola de la provincia de Napo, Ecuador, en el periodo 2014 y 2015.

Table 6. Total number of leaves and proportion of green, yellow, and dry leaves of I. spectabilis, in Arosemena Tola, Napo province, Ecuador, from 2014 to 2015.

\begin{tabular}{ccccc}
\hline $\begin{array}{c}\text { Días desde la } \\
\text { emergencia }\end{array}$ & $\begin{array}{c}\text { Número de hojas totales } \\
\text { por planta promedio* }\end{array}$ & Verdes & Proporción de hojas (\%) \\
\cline { 3 - 5 } & 1,90 a $(4,71)$ & 100 & 0 & 0 \\
\hline 30 & 3,34 b $(11,92)$ & 99,7 & 0,3 & 0 \\
120 & 3,71 bc $(14,92)$ & 97,5 & 1,1 & 1,4 \\
210 & 4,17 c $(18,30)$ & 100 & 0 & 0 \\
300 & EE $( \pm$ ) $\mathbf{0 , 2 1}$ & & & \\
\hline
\end{tabular}

* Valores entre paréntesis son el promedio de número de hojas por conteo. Datos transformados según $\sqrt{ } \mathrm{X}$ para número total de hojas. a,b,c Valores con letras comunes no difieren para $\mathrm{P}<0,05$ (Duncan, 1955) / Values in parentheses are the average number of leaves per count. Data transformed according to $\sqrt{ } \mathrm{X}$ for total number of leaves. $\mathrm{a}, \mathrm{b}, \mathrm{c}$ Values with common letters do not differ for $\mathrm{P}<0.05$ (Duncan, 1955). 
Cuadro 7. Longitud de ramas en la especie I. spectabilis, en el cantón Arosemena Tola de la provincia de Napo, Ecuador, en el periodo 2014 y 2015.

Table 7. Branch length of I. spectabilis, in Arosemena Tola, Napo province, Ecuador, from 2014 to 2015.

\begin{tabular}{|c|c|}
\hline Días desde la emergencia & Largo de ramas promedio $(\mathrm{cm})$ \\
\hline 30 & $3,11 \mathrm{a}$ \\
\hline 120 & $8,24 \mathrm{a}$ \\
\hline 210 & $14,74 \mathrm{~b}$ \\
\hline 300 & $20,75 \mathrm{c}$ \\
\hline \multicolumn{2}{|c|}{ Valor de $P<0,0001 \quad$ EE $( \pm) 1,96$} \\
\hline
\end{tabular}

a, b, c Valores con letras comunes no difieren para $\mathrm{P}<0,05$ (Duncan, 1955) / Values with common letters do not differ for $\mathrm{P}<0.05$ (Duncan, 1955).

Cuadro 8. Número total de hojas, proporción de hojas verdes, amarillas y secas de la especie I. edulis, en el cantón Arosemena Tola de la provincia de Napo, Ecuador, en el periodo 2014 y 2015.

Table 8. Total number of leaves and proportion of green, yellow, and dry leaves of I. edulis, in Arosemena Tola, province, Ecuador, from 2014 to 2015 .

\begin{tabular}{ccccc}
\hline \multirow{2}{*}{$\begin{array}{c}\text { Días desde la } \\
\text { emergencia }\end{array}$} & $\begin{array}{c}\text { Número de hojas } \\
\text { promedio* }\end{array}$ & Verdes & Porcentaje de hojas (\%) \\
\cline { 3 - 5 } & $2,39 \mathrm{a}(6,02)$ & 100 & Amarillas & Secas \\
\hline 30 & $2,90 \mathrm{a}(8,93)$ & 100 & 0 & 0 \\
120 & $3,76 \mathrm{~b}(15,91)$ & 95,4 & 0 & 4,6 \\
210 & $4,55 \mathrm{c}(22,93)$ & 91,54 & 3,68 & 4,78 \\
300 & & & \\
\hline
\end{tabular}

* Valores entre paréntesis son el promedio de número de hojas por conteo. Datos transformados según $\sqrt{ } \mathrm{X}$ para número total de hojas. $\mathrm{a}, \mathrm{b}, \mathrm{c}$ Valores con letras comunes no difieren para $\mathrm{P}<0.05$ (Duncan, 1955) / Values in parentheses are the average number of leaves per count. Data transformed according to $\sqrt{\mathrm{X}}$ for total number of leaves. a, b, c Values with common letters do not differ for $\mathrm{P}<0.05$ (Duncan, 1955).

La especie Inga edulis (Cuadro 9), comenzó a desarrollar sus yemas laterales a partir de los 25 días desde la emergencia, y tuvo a los 30 días media de $3,56 \mathrm{~cm}$, con diferencias a los 300 días.

Cuadro 9. Longitud de ramas de la especie I. edulis, en el cantón Arosemena Tola de la provincia de Napo, Ecuador, en el periodo 2014 y 2015.

Table 9. Branch length of I. edulis. in Arosemena Tola, Napo province, Ecuador, from 2014 to 2015

\begin{tabular}{cc}
\hline Días desde la emergencia & Longitud promedio de ramas $(\mathbf{c m})$ \\
\hline 30 & $3,56 \mathrm{a}$ \\
120 & $7,47 \mathrm{a}$ \\
210 & $16,42 \mathrm{a}$ \\
300 & $36,45 \mathrm{~b}$ \\
\hline & Valor de $\mathrm{P}<0,0001 \mathrm{EE}( \pm) 4,49$
\end{tabular}

a, b Valores con letras comunes no difieren para $\mathrm{P}<0,05$ (Duncan 1955) / Values with common letters do not differ for $\mathrm{P}<0.05$ (Duncan 1955). 


\section{Discusión}

Describir las características del crecimiento de una especie, permite su comparación en diferentes condiciones climáticas e identificar si en la zona de estudio su comportamiento es más promisorio o no, y considerarlo dentro de las estrategias para reinserción o producción.

El comportamiento descrito para la altura de la planta en E. stipitata en esta investigación, corrobora que es una especie de crecimiento lento (Do-Nascimento y De-Oliveira, 1999), y puede alcanzar los 1,1 m a los dos años de edad. Esta característica dificulta su supervivencia en medios silvestres por competencia, requiriendo para su desarrollo, labores continuas de mantenimiento. Al tener esta especie principalmente usos en producción de fruta, la característica de crecimiento es un aspecto a considerar para productores de la zona. Pardos (1985) plantea que, el crecimiento polinomial de primer orden puede ajustarse a cortos períodos de tiempo en las especies vegetales. Esto puede justificar los resultados obtenidos en E. stipitata, donde se observaron comportamientos lineales en el crecimiento de la altura de la planta y diámetro, indicando a su vez que la planta no comenzó su fase de crecimiento exponencial. Es una especie que ramifica cerca del suelo (Escobar et al., 1996), y a los doce meses la planta puede tener entre 25 a 100 $\mathrm{cm}$ de crecimiento lateral (Do-Nascimento y De-Oliveira, 1999). En el presente estudio los valores alcanzados fueron superiores a los informados por estos autores, lo cual puede deberse a características de la zona.

Investigaciones desarrolladas en Centroamérica (CATIE, 1994) demostraron que I. spectabilis, bajo condiciones climáticas diferentes a las de esta investigación, alcanzó alturas entre 17,23 y 21,85 cm a los tres meses de edad y entre 21,91 y $24,96 \mathrm{~cm}$ a los seis meses. Los resultados del presente estudio indicaron similar altura a los tres meses $(19,46 \mathrm{~cm})$ y superior a los seis meses $(32,37 \mathrm{~cm})$, lo que podría indicar la influencia del factor ambiental sobre el crecimiento, donde las precipitaciones influyeron en el mayor crecimiento de las plantas.

La altura a los 320 días de las especies I. spectabilis $(74,13 \mathrm{~cm})$ e I. edulis y $(59,15 \mathrm{~cm})$, así como el desarrollo de sus ramas laterales, permite considerarlas como promisorias, considerando su uso en reforestación o como generadoras de sombra para otros cultivos. En este aspecto Falcao y Clement (2000), plantearon que, I. edulis a edades entre tres y cuatro años pueden alcanzar alturas aproximadas de 4 a $5 \mathrm{~m}$ de altura y 3 a $4 \mathrm{~m}$ de copa. También, en cuanto a la producción de hojas, Marín et al. (2012) en Quindio Colombia, indicaron que en I. edulis, no fue evidente la caída de hojas, teniendo una generación continua y bajos porcentajes de caída de las hojas, generándose mayores brotes en las épocas de picos lluviosos. Los resultados del presente trabajo mostraron mayores porcentajes de hojas amarillas y secas a los 300 días, 3,68 y 4,78, respectivamente, período en el cual comenzó a presentarse menores precipitaciones, lo cual comprueba lo planteado por Marín et al. (2012). Al contrario de esto, Monro et al. (2016) señalaron que, I. edulis presentan hojas de vida corta que se descomponen rápidamente, pero esta característica se presenta a los dieciocho meses de edad.

En función de los resultados y de acuerdo con lo planteado por Cobas-López et al. (2003), en el género Inga, el crecimiento inicial se desarrollaría hasta los 240 días y a partir de ese momento comenzaría la etapa de crecimiento rápido o exponencial, lo cual, de acuerdo con Villar et al. (2005), se ve favorecido por una buena disposición de agua y nutrientes, por lo cual, la presencia de precipitaciones en el presente trabajo influyó en este desarrollo. Estas características de crecimiento indican que, las dos especies del género Inga pueden ser utilizadas en la zona para diferentes usos, tanto en sistemas agroforestales como para reinserción en el medio natural.

\section{Conclusiones}

Los resultados demostraron que las especies E. stipitata, I. edulis e I. spectabilis presentaron diferencias en el crecimiento durante los primeros 320 días posteriores a la siembra. 
I. spectabilis e I. edulis tuvieron crecimiento rápido, mientras que, E. stipitata mostró menores tasas de crecimiento de altura de la planta y diámetro del tallo. Esto potencializa a las dos primeras para su inserción en sistemas agroforestales o naturales, pudiendo concluir que se encontraban terminando su etapa de crecimiento inicial y empezando la fase de crecimiento exponencial en el periodo evaluado, y dados los usos que se reportan en la bibliografía, permitirían diversificar la producción en la zona.

La mayor presencia de hojas verdes en todas las etapas de medición, teniendo bajos porcentajes de hojas amarillas y secas, son también indicadores de que las especies se encontraban en etapa de crecimiento vegetativo.

El análisis de varianza de dos vías permitió identificar que los factores edad de la planta y precipitación, influyeron en la altura de la planta y diámetro del tallo, principalmente en las especies del género Inga.

\section{Literatura citada}

Baruch, Z., y M. Fisher. 1991. Factores climáticos y de competencia que afectan el desarrollo de la planta en el establecimiento de una pastura. En: C.E. Lascano, y J. Spain, editores, Memorias de la VI Reunión del Comité Asesor de la Red Internacional de Evaluación de Pastos Tropicales, Establecimiento y renovación de pasturas: conceptos, experiencias y enfoque de la investigación. CIAT, Cali, COL. p. 103-142.

Cobas-López, M., R. Sotolongo-Sospedra, I. García-Corona, I. Estévez-Valdés, y E. González-Izquierdo. 2003. Comportamiento del crecimiento en altura de Hibiscus elatus Sw. cultivada en contenedores. Rev. Chapingo Ser. Cienc. For. Ambiente 9:131-135

Cordero, J., y D.H. Boshier (ed.). 2003. Árboles de Centroamérica: un manual para extensionistas. CATIE, Turrialba, CRC.

CATIE. 1993. Informe final: proyecto árboles fijadores de nitrógeno. CIID, y CATIE, Turrialba, CRC.

Di-Benedetto, A., y J. Tognetti. 2016. Técnicas de análisis de crecimiento de plantas: su aplicación a cultivos intensivos. Rev. Investig. Agropecu. 42(3):258-282.

Di-Rienzo, J., F. Casanoves, M. Balzarini, L. Gonzalez, M. Cuadroda, y C. Robledo. 2014. InfoStat Software estadístico. InfoStat, ARG. http://www.infostat.com.ar (consultado 01 jun. 2015).

Díaz-Maroto, I.J., A. Prunell, M.P. González, y F.J. Silva. 1993. Estudio fenológico de especies arbustivas y arbóreas de los robledales atlánticos. Ministerio de Agricultura y Pesca, Alimentación y Medio Ambiente, ESP. http://secforestales.org/ publicaciones/index.php/congresos_forestales/issue/view/281 (consultado set. 2017)

Díaz, A., V. Torres, S. Herrera, L. Fernández, y L. Sarduy. 2014. Modelación de crecimiento de bovinos en pastoreo con gramíneas y leguminosas. Zootec. Trop. 32:363-376.

Do-Nascimento, S., y F. De-Oliveira. 1999. Arazá (Eugenia stipitata) cultivo y utilización. FAO. http://www.otca.info/portal/ admin/_upload/publicacoes/SPT-TCA-VEN-SN-araza.pdf. (consultado 5 may. 2016).

Duncan, D.B. 1955. Multiple range and multiple F test. Biometrics 11:1-42. doi:10.2307/3001478

Escobar, C.L., J.J. Zuluzaga, y A. Martínez. 1996. El cultivo de arazá (Eugenia stipitata). CORPOICA, Caquetá, COL.

Falcao, M., e C.R. Clement. 2000. Fenologia e produtividade do Infá-Cipó (Inga edulis) na Amazônia Central. Acta Amaz. 30:173-180. doi:10.1590/1809-43922000302180

Geilfus, F. 1994. El árbol al servicio del agricultor, Manual de agroforestería para el desarrollo rural. 2a ed. ENDA CARIBE/ CATIE, Turrialba, CRC. 
Gobierno-Autónomo-Descentralizado-Provincial-de-Pastaza. 2015. Reseña histórica Santa Clara. Pastaza, ECU. http://www. pastaza.gob.ec/pastaza/santa-clara (consultado 5 may. 2015).

Grime, J.P. 2006. Plant strategies, vegetation processes, and ecosystem properties. $2^{\text {nd }}$ ed. John Wiley \& Sons, Bafins-Lane, GBR.

Hernández, M.S., J.A. Barrera, J.P. Fernández-Trujillo, M.P. Carrillo, y X.L. Bardales. 2007. Manual de manejo de cosecha y postcosecha de frutos de Arazá (Eugenia stipitata Mc. Vaught) en la Amazonía colombiana. Instituto Amazónico de Investigación Científicas SINCHI, Bogotá, COL.

Herrera, J., R. Alizaga, E. Guevara, y V. Jiménez. 2006. Germinación y crecimiento de la planta. Editorial Universidad de Costa Rica, San José, CRC.

Herrera, M. 2013. Métodos estadísticos alternativos de análisis con variables discretas y categóricas en investigaciones agropecuarias. Tesis Dr., Universidad Agraria de La Habana, San José, CUB.

IBM (International Business Machines). 2013. IBM SPSS Statistics. version 22.0 for Windows. IBM Corporation, NY, USA.

Marín, O.H., A.F. Castaño, y G.D. Gómez. 2012. Fenología del guamo Inga edulis (Fabales: Mimosoideae) en dos agroecosistemas del Quindío, Colombia. Rev. Invest. Univ. Quindío 23(2):127-133.

Mariscal, A. 2016. Local baseline knowledge for conservation and restoration of degraded ecosystems in Ecuador. Thesis Dr., Southern Swedish Forest Research Centre, Alnarp, SUE.

Medeiros, J.R., N. Medeiros, H. Medeiros, L.B. Davin, and N.G. Lewis. 2013. Composition of the bioactive essential oils from the leaves of Eugenia stipitata McVaugh ssp. sonoria from the Azores. JEOR 15:293-295. doi:10.1080/10412905.2003. 9712145

Monro, A., R. Velarde, R. Flores, V. Soruco, J. Reyes, y W. Miliken. 2016. Manual agroforestería Inga. Royal Botanic Gardens, Kew \& Herencia, Chicago, IL, USA.

Pardos, J. 1985. Fisiología vegetal. Escuela Técnica Superior de Ingenieros de Montes Fundación Conde del Valle de Salazar, Madrid, ESP.

Proyecto-Manejo-Sostenible-de-Recursos-Naturales. 2007. Manual de agroforestería. Proyecto Manejo Sostenible de Recursos Naturales. San Lorenso, PAR. http://www.mag.gov.py/bina/dato/Manual\%20de\%20agroforesteria.pdf (consultado 5 mayo 2017).

Ríos, M., M.J. Koziol, H. Borgtof, y G. Granda (eds.). 2007. Plantas útiles del Ecuador: aplicaciones, retos y perspectivas. Abya Yala, Quito, ECU.

Rodríguez, L. 2015. Modelación y simulación de la producción de biomasa de Pennisetum purpureum Schum vc. king grass y su aplicación en la alimentación animal. Tesis Dr., Universidad Agraria de La Habana, San José, CUB.

Rodríguez, W., y D. Leihner. 2006. Análisis del crecimiento vegetal. Editorial Universidad de Costa Rica, San José, CRC. https://books.google.com.ec/books?id=69570TbkSxwC\&lpg=PR6\&ots=z1 ry2cx6YV\&dq=Rodr\%C3\%ACqu ez $\% 20 \mathrm{An} \% \mathrm{C} 3 \%$ A 1 lisis $\% 20 \mathrm{de} \% 20 \mathrm{crecimiento} \% 20 \mathrm{vegetal} \& \mathrm{hl}=\mathrm{es} \& \mathrm{pg}=\mathrm{PP} 1 \# \mathrm{v}=$ onepage $\& \mathrm{q}=\mathrm{Rodr} \% \mathrm{C} 3 \% \mathrm{ACquez} \% 20$ An\%C3\%A1lisis\%20de\%20crecimiento\%20vegetal\&f=false (consultado 5 may. 20016).

Ruiz, T., V. Torres, G. Febles, H. Díaz, and J. González. 2013. Growth performance of ecotypes of Tithonia diversifolia according to morphological components. Liv. Res. Rural Dev. 25:9. http://www.lrrd.org/lrrd25/9/ruiz25154.html (accessed 5 May. 20016).

SENPLADES (Secretaría nacional de Planificación y Desarrollo). 2015. Agenda Zonal ZONA 2-Centro Norte; provincias Pichincha Napo y Orellana. Secretaría Nacional de Planificación y Desarrollo, Quito, ECU. 
Statpoint. 2005. Software estadístico Statgraphics Plus. versión 5.1 para Windows. Statpoint, Warrengtong, VA, USA.

Salazar, R., C. Soihet, y J. Méndez. 2000. Manejo de semillas de 100 especies forestales de América Latina. CATIE, Turrialba, CRC.

Systat. 2002. Software estadístico Table Curve 2D version 5.01 para windows. Systat, Chicago, IL, USA.

Villar, R., J. Ruiz, J.L. Quero, H. Poorter, F. Valladares, y T. Marañón. 2005. Tasa de crecimiento en especies leñosas: aspectos funcionales e implicaciones ecológicas. En: F. Valladares, editor, Ecología del bosque mediterráneo en un mundo cambiante, EGRAF S.A., Madrid, ESP. p. 193-230. http://digital.csic.es/handle/10261/47933 (consultado 5 may. 2016).

Vilchez, J., L. Martínez, y N. Albany. 2015. Comparación del crecimiento en vivero entre plántulas y vitroplantas de guayabo cultivar enana roja cubana Eea-1840. Interciencia 14(4):270-274. 